\title{
ON THE ROLE OF d-d ELECTRON CORRELATIONS IN THE COHESION AND FERROMAGNETISM OF TRANSITION METALS
}

\author{
J. FRIEDEL \\ Laboratoire de Physique des Solides (*), Université Paris-Sud, 91405 Orsay, France \\ and \\ C. M. SAYERS \\ Imperial College, Physics Department, London SW7 2AZ, UK
}

(Reçu le 21 décembre 1976, accepté le 16 février 1977)

\begin{abstract}
Résumé. - Le rôle des corrélations d-d dans la stabilité des phases des métaux de transition est étudié d'une façon semi-quantitative, en utilisant une formule de perturbation du second ordre qui peut être reliée à l'approximation de Gutzwiller. Cette déviation par rapport à l'approximation de Hartree-Fock augmente la cohésion et réduit la tension superficielle ; elle déstabilise le ferromagnétisme, particulièrement pour des bandes $d$ presque à moitié pleines. L'effet maximum sur la cohésion et la tension superficielle se produit pour une bande $d$ à moitié pleine ; il reste petit, au moins pour les séries $4 d$ et $5 d$.

Abstract. - The role of $d-d$ correlations in the stability of phases in transition metals is studied in a semi-quantitative way, using a second-order perturbation formula which can be related to Gutzwiller's approximation. This deviation from the Hartree-Fock approximation increases the cohesion and decreases the surface tension; it destabilizes ferromagnetism, especially for nearlyhalf-full $\mathrm{d}$ bands. The maximum effect on cohesion and surface tension occurs for a half-filled $\mathrm{d}$ band; it remains small, at least for the $4 \mathrm{~d}$ and $5 \mathrm{~d}$ series.
\end{abstract}

1. Introduction. - The purpose of this paper is to analyse, in a simple and semi-quantitative way, the possible effect of correlations between $\mathrm{d}$ electrons on the cohesive and magnetic properties of transition metals.

To show the type of effects expected and estimate their order of magnitude, we shall use as a starting point a simple tight binding (Huckel) description of the $d$ band [1], and then include $d$ electron correlations as a perturbation. We assume that the number of $\mathrm{s}$ electrons per atom remains a constant near to unity and only adds a small constant term to the cohesion. We shall also neglect secondary effects such as interatomic electrostatic [2], Van der Waals [3], and exchange terms [4] and ds mixing [5]. We shall introduce the spin-orbit coupling $\lambda$ I.s only to first order in perturbation [6], in which case it plays a role in the energy of free atoms but not in that of the metals, where the orbital moment is frozen to first order [7]. We shall restrict ourselves to a simplified picture of the $\mathrm{d}$ one-electron band, where all five $\mathrm{d}$ orbitals with different orbital moments $(m=2$ to -2$)$ are assumed

(*) LA du C.N.R.S. equally populated at each energy (Fig. 1), and the total energy band is assumed symmetrical with respect to the atomic energy and rectangular. Then the density of states $n(E)$ per atom is assumed to be (Fig. 2)

$$
\begin{aligned}
& n=\text { const }=10 / w \quad \text { for }-\frac{1}{2} w \leqslant E \leqslant \frac{1}{2} w \\
& n=0 \text { elsewhere }
\end{aligned}
$$

where $w$ is the width of the band. These two last assumptions are known to give a reasonable starting

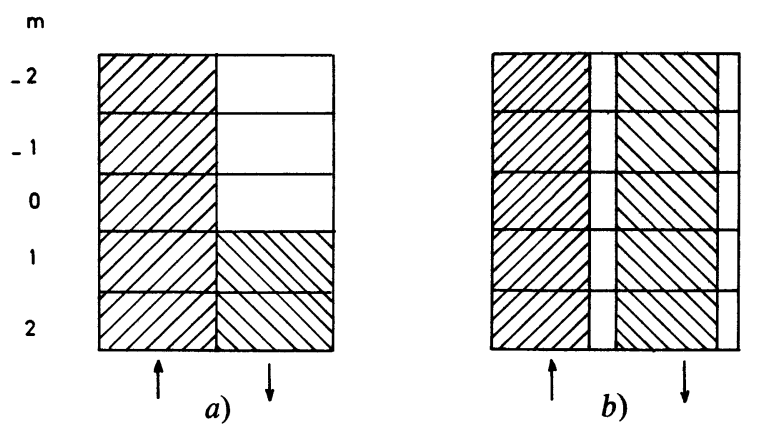

Fig. 1. - Assumed filling of the $\mathrm{d}$ atomic orbitals : $a$ ) free atom; b) paramagnetic metal. 


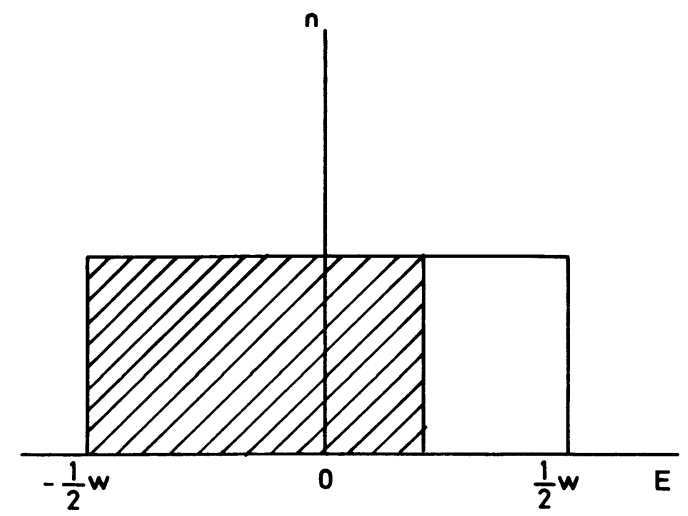

Fig. 2. - Assumed form of the $d$ band.

point [1], $[8,9,10]$ for an order of magnitude estimate of cohesive properties. There would be no a priori difficulty in improving on them.

Finally we shall describe the correlations in terms of average intra-atomic Coulomb and exchange interactions $U$ and $J$. $U$ is an average value, taken over all possible couples of $\mathrm{d}$ orbitals; $J$ is the usual average exchange energy term : it is the difference in energy between two electrons of antiparallel spins, so that $2 J$ is the difference in energy between the atomic triplet and singlet states [1], [4].

In most of this discussion, we shall assume the parameters $w, U, J$ and $\lambda$ to take constant values in a given series, independent of the number $z$ of $d$ electrons per atom in the corresponding $\mathrm{d}$ band.

Most of the approximations listed above can be improved, and their relative importance checked. This should certainly be done in a detailed quantitative comparison with experiment, and indeed various attempts have been made within the Hartree or Hartree-Fock scheme [11]. We shall actually relax the assumption of constant parameters $w, U$ and $J$ in the discussion of magnetism at the end of this paper. But, to give a semi-quantitative estimate of the role of correlations in integral quantities such as phase stabilities, it seems that these rough assumptions provide accurate enough orders of magnitude.

2. Cohesive energy of a paramagnetic metal. Within the above assumptions, the energy of cohesion per atom $E_{\mathrm{c}}$ is obtained by comparing the electronic energies in free atoms and in the metal. A development in successive powers of $U$ and $J$ gives :

$$
E_{\mathrm{c}}=E_{\mathrm{c}}^{0}+E_{\mathrm{c}}^{1}+E_{\mathrm{c}}^{2}+O\left(U^{3}, U J, U \lambda, J^{2}, \lambda^{2}\right),
$$

where $\left({ }^{1}\right)$

$$
\begin{aligned}
& E_{\mathrm{c}}^{0}=-\int_{\mathrm{M}}^{E_{\mathrm{M}}} n(E) E \mathrm{~d} E \simeq \frac{z(10-z)}{20} w \\
& E_{\mathrm{c}}^{1}=-\frac{z(10-z)}{20} U-2 S(2 S-1) \frac{J}{4}-\lambda L S
\end{aligned}
$$

(1) Expression (4) is only valid for integral values of $z$. For $0<c=z-l<1$ ( $l$ integer), one should add $\frac{1}{2} c(1-c)(U-J)$. This insures that there is no contribution from $J$ unless $z \geqslant 2$. with

$$
\begin{aligned}
2 S & =z & & \\
L & =\frac{1}{2} z(5-z) & & \text { for } z \leqslant 5 ; \\
2 S & =10-z & & \text { for } z \geqslant 5 . \\
L & =\frac{1}{2}(10-z)(z-5) & &
\end{aligned}
$$

Finally

$$
E_{\mathrm{c}}^{2} \simeq \frac{45}{w}\left[\frac{z}{10}\left(1-\frac{z}{10}\right) U\right]^{2}
$$

2.1 ZERO-ORDER BAND TERM. - In these expressions, $E_{\mathrm{c}}^{0}$ is the usual zero-order term, where the zero of energy is taken as that of the localized states in the atomic potentials which build up the crystal potential, so that the zero-order (one electron) hamiltonian only contains the transfer integral terms connecting $d$ orbitals on neighbouring sites. To zero approximation in $U$ and $J$, one can for instance assume that these are the inner ion potentials, or any other potentials which count in the same way the intra-atomic electron interactions in the metal and in the free atoms. From that point of view, it is more natural to take the potentials due to the singly ionized transitional ions [1].

The Fermi energy $E_{\mathrm{M}}$ is related to the number $z$ of $d$ electrons per atom by

$$
z=\int^{E_{\mathrm{M}}} n(E) \mathrm{d} E
$$

The second expression of $E_{\mathrm{c}}^{0}$ in (3) varies parabolically with $z$, with a maximum equal to $\frac{5}{4} w$ for $z=5$ (Fig. $3 a$ ). It is strictly valid for the band of figure 2 ; but a systematic use of equations (3) and (7) and of the moments of $n(E)$ shows that $E_{\mathrm{c}}^{0}$ never deviates very much from this parabolic approximation [1], [10] : it is not very sensitive to the detailed variation of $n(E)$, because it is an integral property of $n(E)$.

2.2 FirST-ORDER HARTREE-FoCK CORRECTION. The Hartree-Fock correction $E_{\mathrm{c}}^{1}$ is obtained by keeping the $\mathrm{d}$ electrons distributed at random over all atomic $\mathrm{d}$ orbitals, but taking into account the Pauli principle, which forbids two electrons with parallel spins to sit at the same time in any of the orbitals [20]. One assumes all ten d spin-orbitals to be equally populated in the paramagnetic metal, but some orbitals of a given spin direction to be preferentially populated in the free atom (Fig. $1 a, b$ ).

Due to the Pauli principle, couples with the same orbital appear with a relative weight equal to half that of couples with different orbitals. In atoms, the Coulomb interaction energy is $\frac{1}{2} z(z-1) U$ : each of the $z$ electrons sees $z-1$ other electrons; the interactions must not be counted twice. In a paramagnetic metal on the other hand, the corresponding term is $\frac{1}{2} \frac{9}{10} z^{2}$ : each of the $z$ electrons will see $\frac{9}{10} z$ electrons of different spin-orbitals; again the inter- 

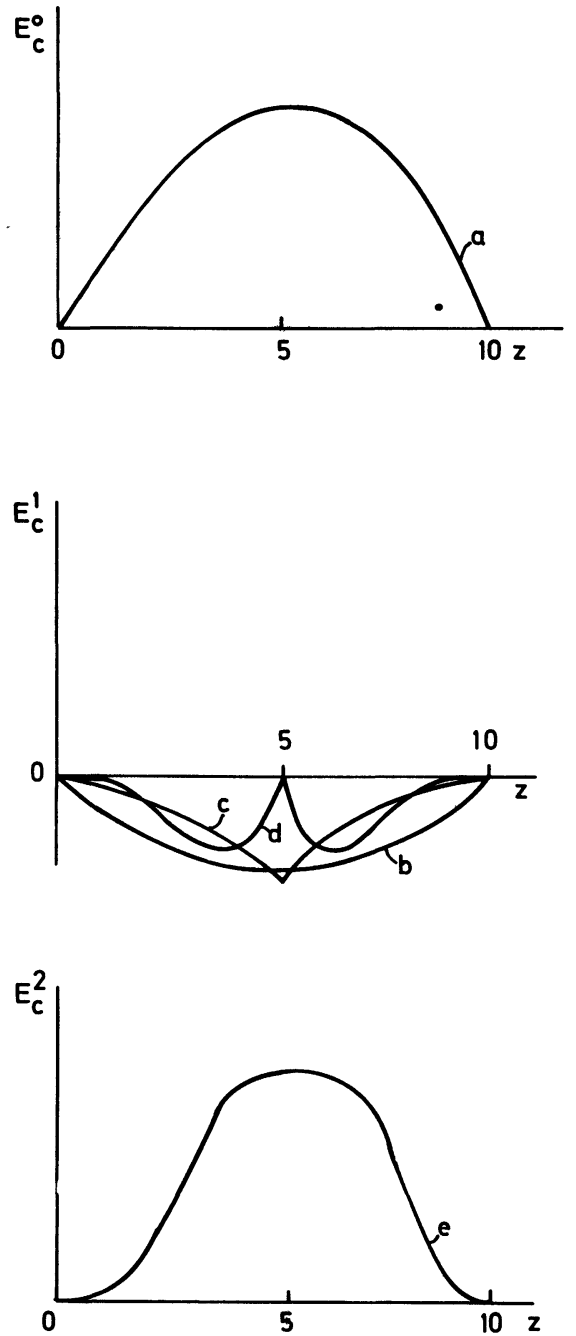

FIG. 3. - Qualitative variation of the various contributions to the cohesive energy in paramagnetic metals : a) band term $E_{\mathrm{c}}^{\mathbf{0}}$-Hartree-Fock corrections $\left.E_{\mathrm{c}}^{1} ; b\right)$ Coulomb $U ; c$ ) exchange $J ; d$ ) spin orbit $\lambda ; e)$ Coulomb correlation correction $E_{\mathrm{c}}^{2}$.

actions must not be counted twice. The corresponding difference in energy contributes to the cohesive energy the parabolic negative term $-\frac{z(10-z)}{20} U$, which reduces the amplitude of the cohesive energy without altering its form (Fig. 3b).

The exchange energy also appears in $E_{\mathrm{c}}^{1}$ because the atom is magnetic and the metal is not (Fig. 1). It produces a central cusp in $E_{\mathrm{c}}^{1}(z)$ for $z=5$, which corresponds to the special stability of the half filled $\mathrm{d}$ atomic shells, due to a maximum in their exchange interactions (Fig. 3c).

The spin-orbit coupling gives no first-order term in the metal, whether paramagnetic or ferromagnetic, in the approximate model used here where the five $d$ orbitals are equally populated (Fig. $1 b$ ). In atoms, it introduces a first-order correction to the energy which, in the $L S$ coupling, is equal to $-\lambda L S$, where the total orbital and spin moments $L$ and $S$ vary with $z$ as given in (5). The spin orbit correction (Fig. 3d) is thus also negative. It introduces a central positive cusp in $E_{\mathrm{c}}$ which essentially broadens that due to the exchange term; this broadening can even lead to a central positive cusp for $\lambda / J>9 / 25$; however the assumed Russel Saunders coupling becomes less valid in that range, and a more refined analysis should be made in this case, for example in the $5 \mathrm{~d}$ series.

2.3 SECOND-ORDER COULOMB CORRELATION TERM. - $E_{\mathrm{c}}^{2}$ is the first correction due to deviations from the Hartree-Fock scheme, owing to interatomic Coulomb correlations. It restricts the atomic charge fluctuations from what they would be for uncorrelated $d$ electrons [20]. Within second-order perturbations, it can be understood as being due to the virtual excitations of each $\frac{z}{10}$ electron per atom in a given spinorbital, into the empty portions $\left(1-\frac{z}{10}\right)$ of orbitals with the same spin direction. The matrix element of each excitation is $U$; the number of excitations is 45 (cf. appendix); and the energy denominator is of the order of the energy band width $w$.

A denominator exactly equal to $w$ is obtained in a similar expression [12] when developing the cohesive energy of a non-degenerate $s$ band treated in Gutzwiller's approximation [13], using the rectangular band approximation of figure 2 ; thus equation (6) can be considered as a direct extension to five-fold degenerate $\mathrm{d}$ bands of the Gutzwiller approximation.

It can also be remarked that the corrections in $U$ and $U^{2}$ in $E_{\mathrm{c}}$ can be considered as the first terms of a development of an effective Hartree-Fock correction

$$
-\frac{z(10-z) U_{\text {eff }}}{20}
$$

with

$$
\begin{aligned}
U_{\text {eff }}=U-9 \cdot \frac{z}{10} & \left(1-\frac{z}{10}\right) \frac{U^{2}}{w} \simeq \\
& \simeq \frac{U}{1+9 \cdot \frac{z}{10}\left(1-\frac{z}{10}\right) \frac{U}{w}} .
\end{aligned}
$$

This is reminiscent of Kanamori's formula [14] .

$$
U_{\mathrm{K}}=\frac{U}{1-\frac{1}{10} F\left(E_{\mathrm{M}}\right) U}
$$

where

$$
-\frac{1}{10} F\left(E_{\mathrm{M}}\right)=P \frac{1}{10} \int \frac{n(E) \mathrm{d} E}{E_{\mathrm{M}}-E} \simeq \frac{1}{w} \ln \left|\frac{10-z}{z}\right|
$$

for the rectangular band of figure 2. Kanamori's formula however could only be valid for nearly empty bands $(0<z \ll 5)$; it treats more correctly the higher-order terms in $U^{n}$ in single site scatter- 
ing, but neglects interferences in multiple sites scattering.

This discussion clearly shows that the correlation correction $E_{n}^{2}$ given by equation (6) is of the same nature as Kanamori's corrective term. It is positive, thus stabilizing the metal, as indeed is expected. Its variation with $z$ as given by the qualitative argument above or deduced from Gutzwiller's approximation is a sinusoid peaked at $z=5$ (Fig. 3e). Finally the second form of formula (8) predicts, as does Kanamori's formula, a saturation effect for large values of $U / w$, as expected from a repulsive interaction; it is in reasonable agreement with an exact computation of Gutzwiller's approximation for large $U$ 's [12].

3. Surface tension of a paramagnetic metal. From (2) to (6), one easily deduces a surface tension per surface atom [10], [15]

$$
\begin{array}{r}
\gamma \simeq\left\{\frac{z(10-z)}{20} w-\frac{45}{w}\left[\frac{z}{10}\left(1-\frac{z}{10}\right) U\right]^{2}\right\} \times \\
\times \alpha \frac{\delta p}{p}+O\left(U^{3}, U J, U \lambda, J^{2}, \lambda^{2}\right)
\end{array}
$$

where $p$ is the number of neighbours in the volume and $\delta p$ the decrease in the number of neighbours for surface atoms. $\alpha$ is a numerical coefficient between 1 and 2 . This can be physically related to the fact that atoms on the surface have an effective local $d$ band width $w-\delta w \simeq(p-\delta p)^{\alpha} w \cdot \alpha=1$ is obtained by assuming that the jumps of an electron from one atom to its neighbours are uncorrelated; $\alpha=\frac{1}{2}$ is obtained by deducing the width from the second moment of the density of states; this is nearer to reality [9].

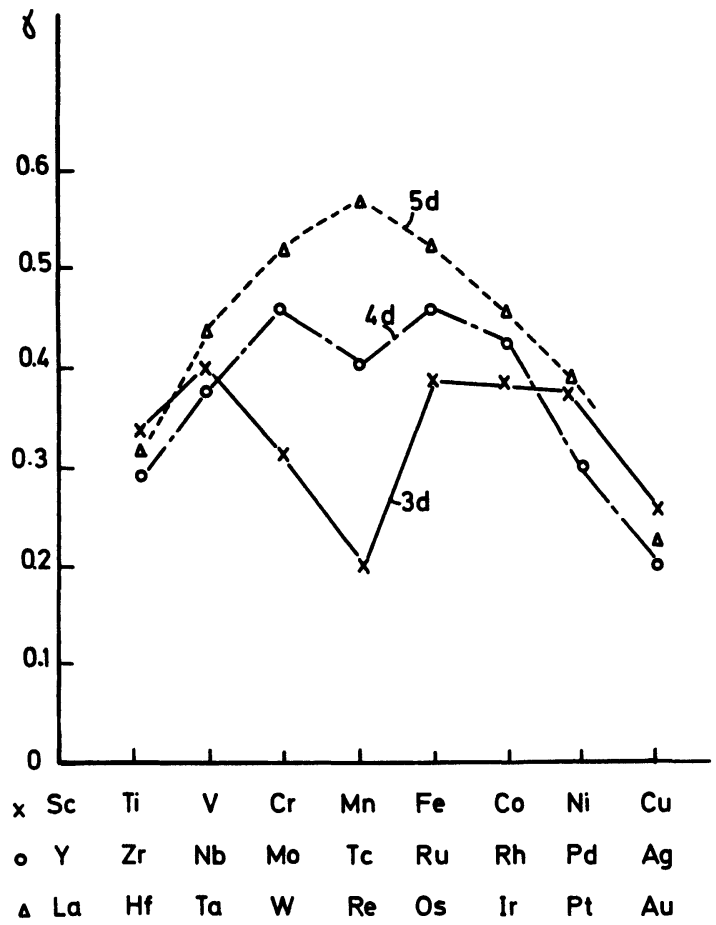

FIG. 4. - Surface tension of liquid transition metals, in $\mathrm{eV}$ per surface atom [15].
Formula (10) shows that the Coulomb correlation correction in $U^{2}$ produces a central dip in the otherwise parabolic variation of $\gamma(z)$ due to the band contribution. Contrary to what happens for the cohesive energy, it appears as a negative correction.

4. Discussion of the cohesive properties of paramagnetic metals. - The surface tension (10) is simpler to discuss than the cohesive energy (2) to (6), because the complex terms in $J$ and $\lambda$ due to the magnetism of free atoms are not involved.

Figure 4 shows that, for liquid transition metals, $\gamma(z)$ is nearly parabolic, at least in the $4 \mathrm{~d}$ and $5 \mathrm{~d}$ series. From the central dip observed in $3 \mathrm{~d}$ and $4 \mathrm{~d}$ series and its absence in the $5 \mathrm{~d}$ one, one deduces values of $U / w$ given by table I. As $U / w<1$, the perturbation scheme used here is fully justified, at least in the $4 \mathrm{~d}$ and $5 \mathrm{~d}$ series. As pointed out above, further terms in the development in $U^{n}$ would only reduce the second-order term.

TABLE I

Values of $w, U, J, \lambda$ in $\mathrm{eV}$

$$
\begin{array}{ccccccccc} 
& U / w & w & U & J & \bar{J} & \bar{\lambda} & \frac{\delta p}{p} & \bar{X} \\
& - & - & - & - & - & - & \frac{1}{c} & - \\
\text { 3d } & (0.5) & (6) & (3) & 0.5 \text { to } 0.9 & 0.7 & 0.05 & 1 / 6 & (0) \\
\text { 4d } & 0.35 & 9 & 3 & 0.4 \text { to } 0.8 & 0.6 & 0.15 & 1 / 8 & -3 \\
\text { 5d } & \leqslant 0.25 & 12 & \leqslant 3 & 0.4 \text { to } 0.75 & 0.55 & 0.40 & 1 / 10 & \leqslant-9
\end{array}
$$

Figure 5 shows in the same way the variation with $z$ of the cohesive energy of the (solid) transition metals, for the phases actually observed at room temperatures. As the latent heats of phase changes are small compar-

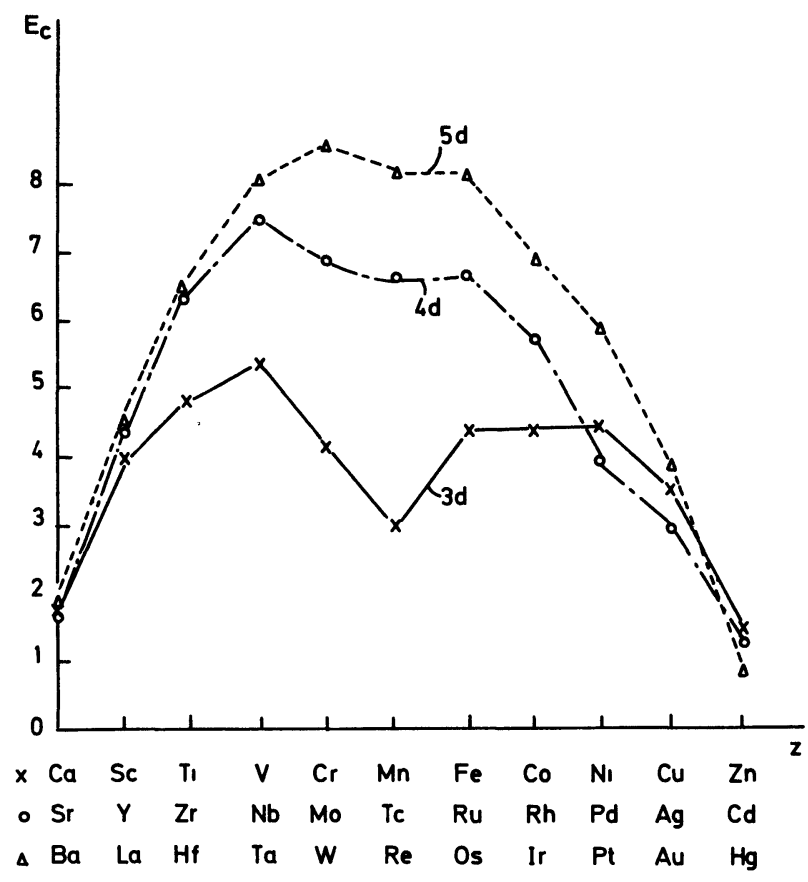

FIG. 5. - Cohesive energy of solid transition metals, in eV per atom [19]. 
ed with these values, the complications arising from differences in crystal structures can be neglected here. To simplify the comparison with equations (2) to (6), it is better to subtract from the measured values of $E_{\mathrm{c}}(z)$ the atomic corrections $-2 S(2 S-1) \frac{J}{4}-\lambda L S$, which are known. Table I gives the range of values of $J$ through each series, and an average value valid near the centre of the series. It also gives an order of magnitude of $\lambda$. Values of the cohesive energies $E_{\mathrm{c}}^{\prime}$ corrected for these atomic terms are given in figure 6 . The correction made here is only approximate, because it uses the average values of $J$ and $\lambda$ of table I and because atoms are assumed to be in the $\mathrm{d}^{n} \mathrm{~s}$ state; also the $L S$ coupling is not well followed in the 5d series. But more exact corrections [11] would not change the essential fact that these corrections remove the central dip in $E_{\mathrm{c}}(z)$, especially in the $4 \mathrm{~d}$ series : the minimum of cohesion in the centre of the series is thus clearly connected with the special stability of the half-filled (magnetic) atomic $\mathrm{d}$ shells. It has a different origin from that observed for $\gamma(z)$, figure 4.

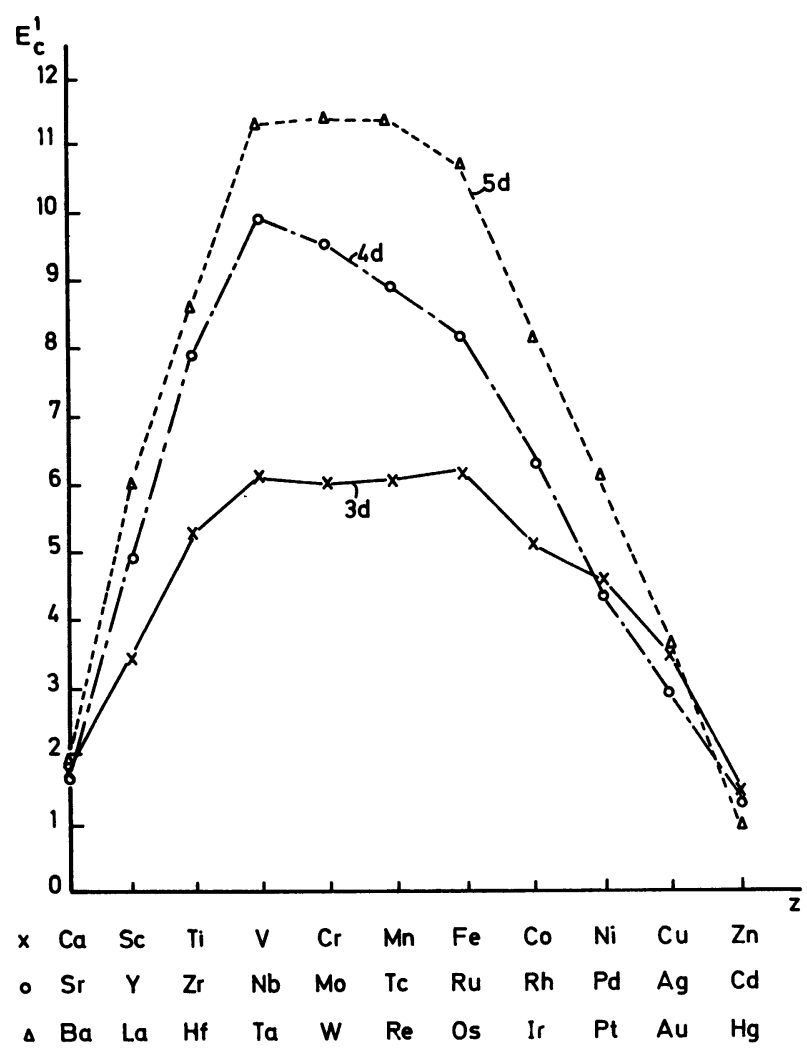

Fig. 6. - Cohesive energy per atom, corrected for the magnetic terms of free atom.

These corrected values of cohesive energy

$$
E_{\mathrm{c}}^{\prime}=E_{\mathrm{c}}+2 S(2 S-1) \frac{J}{4}+\lambda L S
$$

must be compared with the development (equation (2) to (6)) :

$$
E_{\mathrm{c}}^{\prime}=\frac{z(10-z)}{20}(w-U)+\frac{45}{w}\left[\frac{z}{10}\left(1-\frac{z}{10}\right) U\right]^{2}
$$

From the values of $U / w$ deduced table $\mathrm{I}$, one sees that the corrections in $U$ and $U^{2}$ are not too large, especially in the $4 \mathrm{~d}$ and $5 \mathrm{~d}$ series, and somewhat compensate near the middle of the series, where they only reduce cohesion by 10 to $20 \%$. One then deduces from the values of $E_{\mathrm{c}}^{\prime}$ for $z=5$ the values of $w$ and $U$ given in table I. They are reasonable from what one knows about $w$ from $\mathrm{X}$ ray spectra and about $U$ from atomic spectra. The values obtained for $U$ are somewhat small.

The absolute values of $\gamma(z)$, as given by equation (10), then fit with these estimates if we take $\alpha=2$ and $\delta p / p$ equal to $\frac{1}{6}$ to $\frac{1}{10}$ (Table I). A close-packed surface on a close-packed arrangement would lead to $\delta p / p=\frac{1}{4}$. One cannot expect a better order of magnitude agreement, as formula (10) is only a somewhat rough estimate. The difference can in any case be at least partly explained by a more compact arrangement of the liquid at the surface than in the volume, and by surface (vibration and diffusion) entropy terms which probably reduce $\gamma$ by a very appreciable fraction [10].

It is clear that, in the $3 \mathrm{~d}$ series, the corrections due to the Coulomb term $U$ are especially large, and that the fit of $\gamma(z)$ and $E_{\mathrm{c}}^{\prime}(z)$ with the theoretical development in $U$ is especially bad. Thus $\gamma(z)$ has a minimum at $\mathrm{Mn}$ which is sharper than expected from the smooth parabolic correction in $U^{2}$, equation (10); and its variations are not at all symmetrical in $z$ for the two halves of the series. The cohesive energy $E_{\mathrm{c}}^{\prime}$ corrected for the magnetic atomic terms has in the centre of the series a flat plateau, instead of a fairly sharp maximum, as expected from the term in $U^{2}$, equation (12).

As often stated [1], these deviations are probably to be connected with the special magnetic properties of the metals in this series.

We shall restrict the discussion here to the (simpler) problems of ferromagnetism, thus we shall not treat in a proper way the antiferromagnetism of $\mathrm{Cr}$ and $\mathrm{Mn}$. Corrections to the cohesive properties due to the ferromagnetism of the metals will be computed in this same simple model. As expected, they are small enough not to alter very much the overall picture of cohesion, in agreement with the fact that the magnetic order temperatures are well below the melting points. But conversely the Coulomb correlation corrections to cohesion strongly affect the conditions of apparition of magnetism. This is a point well known in the metalinsulator problem [16], but which had not been stressed so much in the context of weaker correlation corrections and transition metals. 
5. Ferromagnetic metals. - We assume now an ordinary band ferromagnetism at $0 \mathrm{~K}$, where

$p=\frac{1}{2}(z+\mu)$ electrons per atom have spins parallel to the magnetic moment

$n=\frac{1}{2}(z-\mu)$ electrons per atom have spins antiparallel to it.

We call $\delta E_{\mathrm{c}}(z, \mu)$ the variation in cohesive energy per atom with $\mu$, at fixed $z$ :

$$
\delta E_{\mathrm{c}}(z, \mu)=E_{\mathrm{c}}(z, \mu)-E_{\mathrm{c}}(z, 0) .
$$

It is easy to see (cf. appendix) that equations (2) to (7) transform so that

$\delta E_{\mathrm{c}}(z, \mu)=\delta E_{\mathrm{c}}^{0}(z, \mu)+\delta E_{\mathrm{c}}^{1}(z, \mu)+\delta E_{\mathrm{c}}^{2}(z, \mu)+\cdots$

with

$$
\begin{aligned}
& \delta E_{\mathrm{c}}^{0}(z, \mu)=-w \mu^{2} / 20 \\
& \delta E_{\mathrm{c}}^{1}(z, \mu) \simeq(U+4 J) \mu^{2} / 20 \\
& \delta E_{\mathrm{c}}^{2}(z, \mu) \simeq \\
& \simeq\left[-100 \mu^{2}-140 z \mu^{2}+14 z^{2} \mu^{2}+9 \mu^{4}\right] \frac{U^{2}}{2000 w}
\end{aligned}
$$

$\delta E_{\mathrm{c}}^{1}(z, \mu)$ is the Hartree-Fock term, which leads to the Stoner criterion [17] for ferromagnetism, applied to the rectangular band considered here.

$\delta E_{\mathrm{c}}^{2}(z, \mu)$ is the correction arising from the change with magnetism of the Coulomb correction term $E_{\mathrm{c}}^{2}$ (equation (6)). As $E_{\mathrm{c}}^{2}(z)$ is positive and a maximum for a half-filled band (Fig. 3e), it is clear that its contribution to cohesion is reduced for metals near to the middle of a series, when, owing to ferromagnetism, the two half $\mathrm{d}$ bands become unequally populated $(p \neq n)$. Indeed, it is clear that the Coulomb correlations contribute a correction to the Stoner criterion of incipient ferromagnetism which is against magnetism near the centre of a transitional series, where $\frac{\partial^{2} E_{\mathrm{c}}^{2}}{\partial z^{2}} \ll 0$ (cf. Fig. $3 e$ ).

More specifically, we call, as usual [1], [20] weak ferromagnetism an incomplete unbalance of spins, so that no spin direction is either totally full or empty. Strong ferromagnetism corresponds to the complete unbalance (Fig. 7).
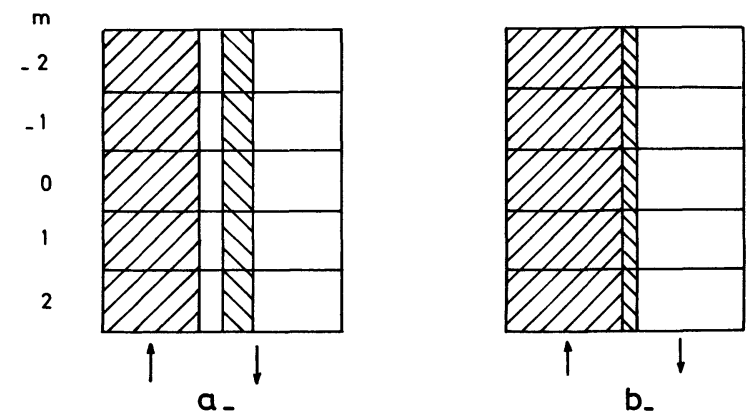

Fig. 7. - Two types of band ferromagnetism : $a$ ) weak; $b$ ) strong.
To first order in $U$ and $J$, the energy difference between a ferromagnetic state of atomic moment $\mu$ and the corresponding paramagnetic state $(\mu=0)$ with the same number $z$ of electrons is

$$
\delta E_{\mathrm{c}}^{1}(\mu)=(U+4 J-w) \frac{\mu^{2}}{20} .
$$

Thus the ferromagnetic state becomes more stable for

$$
U+4 J-w>0 \text {. }
$$

This is Stoner's criterion [17], applied to the present simplified band structure (rectangular band of width $w$, with equal populations of different $\mathrm{d}$ orbitals). When this criterion is fulfilled, and to this order in $U$, strong ferromagnetism should be preferred to weak ferromagnetism, thus $\mu$ should take its maximum possible value $2 S(z)$, as given by (5).

However the second-order correction (17) contributes to the difference in energy between the strong ferromagnetic and paramagnetic states a term

$$
\delta E_{\mathrm{c}}^{2}(z)=\frac{U^{2}}{w} y(z)
$$

where

$$
y(z)=\left(23 z^{2}-140 z-100\right) \frac{z^{2}}{2000}
$$

which is always negative, and strongly peaked at $z=5$ (Fig. 8). This clearly works against ferromagnetism, especially for nearly half filled $\mathrm{d}$ bands, where strong deviations from Stoner's criterion (20) are thus expected.

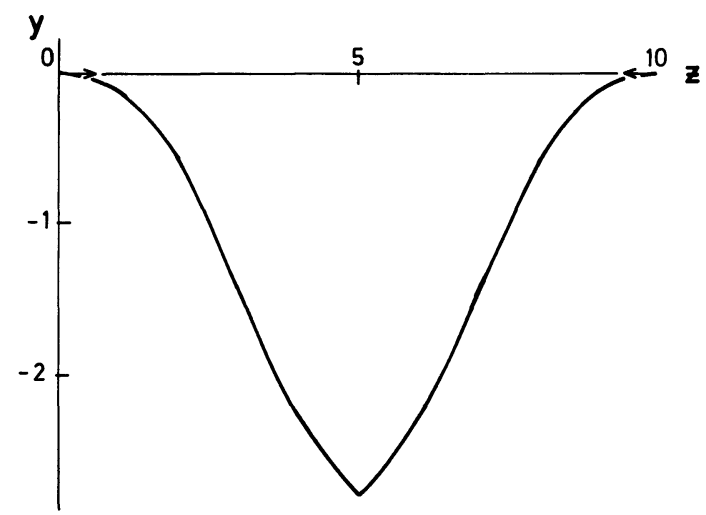

FIG. 8. - Variation of $y(z)$.

Figure 9 shows that $\delta E_{\mathrm{c}}(z, \mu)$ as given by (14) can behave in two different ways. Let us introduce the quantity

$$
X=(U+4 J-w) w / U^{2} .
$$

a) For

$$
X>X_{1}(z)=1+0.14 z(10-z)
$$

$\delta E_{\mathrm{c}}(z, \mu)$ increases parabolically with $\mu^{2}$ for fixed $z$ (Fig. 9a). In this range, the paramagnetic state is 

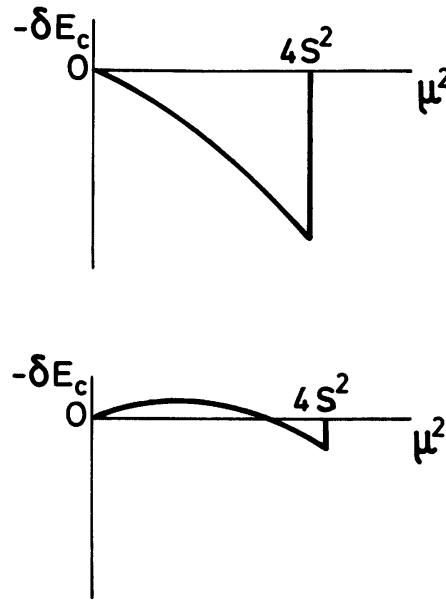

b_

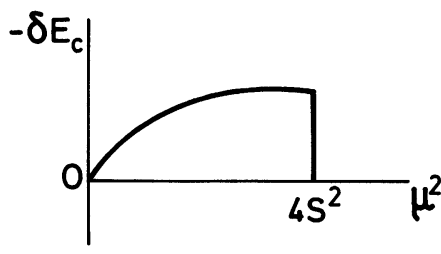

C_

Fig. 9. - Three types of variations of $\delta E_{\mathrm{c}}(z, \mu)$ versus $\mu^{2}$.

unstable and the only stable state is the strong ferromagnetic one, with $\mu=2 S(z)$.

b) For

$$
X<X_{1}(z)
$$

$\delta E_{\mathrm{c}}(z, \mu)$ has still a positive curvature in $\mu^{2}$, but decreases for increasing $\mu^{2}$ for small values of $\mu^{2}$.

Then

$$
\left.b_{1}\right) \text { For }
$$

$$
X<X_{2}(z)=X_{1}(z)-0.09 z^{2}
$$

$\delta E_{\mathrm{c}}(z, 2 S(z))<0$ (Fig. $9 c$ ). The only stable state is the paramagnetic one $\mu=0$.

$b_{2}$ ) But for

$$
X>X_{2}(z)
$$

$\delta E_{\mathrm{c}}(z, 2 S(z))>0$ (Fig. $\left.9 b\right)$. The paramagnetic state $\mu=0$ is metastable. But the most stable state is the strong ferromagnetic one $\mu=2 S(z)$.

Figure 10 compares these new criteria for ferromagnetism with Stoner's criterion $X \gtrless 0$. It confirms that the ferromagnetism is less stable, especially for nearly half filled $\mathrm{d}$ bands. With this simple model, ferromagnetism can however be the stable state even when paramagnetism is metastable; but weak ferromagnetism is never obtained.

The difference between figures $10 a$ and $b$ can be understood in the following way :

- the term in $\mu^{2}$ in $\delta E_{\mathrm{c}}^{2}(z, \mu)$, equation (17) shifts the Stoner criterion to the curve $X_{1}(z)$, thus strongly against ferromagnetism ;

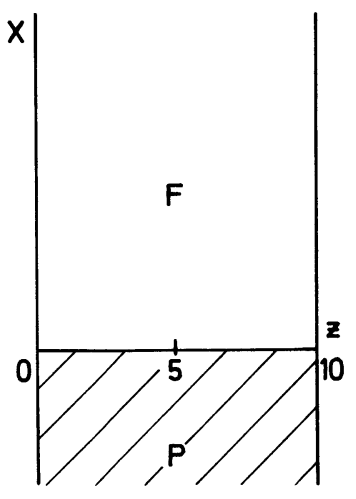

a-

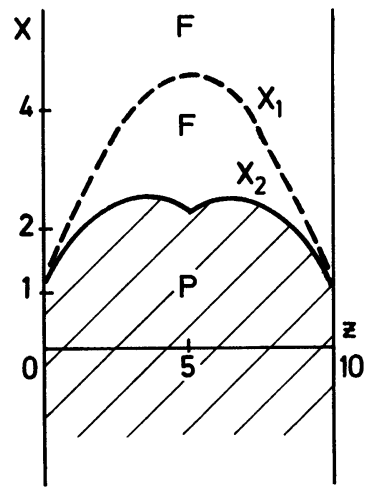

b_
FIG. 10. - Criteria for paramagnetism $\mathbf{P}$ and strong ferromagnetism $\mathrm{F}$ for a rectangular band : $a$ ) Stoner's Hartree-Fock firstorder criterion; $b$ ) second-order corrections in Coulomb correlations added. Between $X_{1}$ and $X_{2}$, the paramagnetic state is metastable.

- however the term in $\mu^{4}$ in $\delta E_{\mathrm{c}}^{2}(z, \mu)$ favours ferromagnetism, and can thus make it more stable than paramagnetism even when this is metastable (region between $X_{1}$ and $X_{2}$ ).

As a result the range of stability of paramagnetism is less peaked towards the positive $X$ region than would have been expected from inspection of figure 8 .

6. Discussion of ferromagnetism. - From the average values of $w, U$ and $J$ deduced above, one would deduce the average values of $\bar{X}$ in the three transitional series given in table $I$.

The Stoner criterion (Fig. 10a) would then predict, according to the sign of $\bar{X}$, that the $3 \mathrm{~d}$ series should be nearly magnetic while the $4 \mathrm{~d}$ and $5 \mathrm{~d}$ should be strongly paramagnetic.

The Coulomb correlation corrections (Fig. 10b) alter this picture somewhat : with the average values of $\bar{X}$ given table I, one would expect the $3 \mathrm{~d}$ series to be strongly paramagnetic too.

This semi-quantitative description should be corrected on several counts :

- through each transition series, $U$ and $J$ increase and $w$ decreases while $z$ varies from 0 to 10 . As a result, $X(z)$ increases with $z$. This certainly helps to explain why ferromagnetism occurs at the end of the second half of the $3 \mathrm{~d}$ series $(\mathrm{Fe}, \mathrm{Co}, \mathrm{Ni})$, and the tendency towards ferromagnetism at the end of the $4 d$ and $5 d$ series $(\mathrm{Pd}, \mathrm{Pt})$;

- the numerical estimates are only orders of magnitude, and can only express general tendencies. Thus the correlation correction, which plays a large role here, is only expressed within a numerical factor of order unity; the rectangular band approximation is but a rough approximation : the peak of $n(E)$ in the upper half of the $d$ bands in cubic phases helps the occurrence of ferromagnetism and the minimum 
of $n(E)$ near the middle of the series certainly plays a role in the occurrence of weak ferromagnetism [1] ; the assumption of equal population of the five $d$ orbitals is especially poor for nearly full bands, and consequently spin orbit corrections are important there in the $4 d$ and $5 d$ series $(\mathrm{Pd}, \mathrm{Pt})$ [6], [1].

The discussion above shows clearly however that the Coulomb correlation correction plays an important role in magnetism :

It works against ferromagnetism in the $3 \mathrm{~d}$ series, and forbids it in the middle of the series; it must be taken into account in the balance of near magnetism for Pd and Pt, at the end of the $4 \mathrm{~d}$ and $5 \mathrm{~d}$ series.

Finally for the observed magnetic phases of the $3 \mathrm{~d}$ series in $\mathrm{Fe}, \mathrm{Co}, \mathrm{Ni}$, we can compute magnetic corrections to the cohesive energy and surface tension. With the values of the parameters of table $I$, we check from equations (14) or (24) that the corresponding changes are less than one $\mathrm{eV}$ for cohesion and a few $0.1 \mathrm{eV}$ for the surface tension. This alters the detailed form of $E_{\mathrm{c}}(z)$ and $\gamma(z)$, without changing the general roughly parabolic variation of $E_{\mathrm{c}}(z)$. It is clear that a discussion of the stability of antiferromagnetism is required to draw a full conclusion on the behaviour of $E_{\mathrm{c}}(z)$ and $\gamma(z)$ in the $3 \mathrm{~d}$ series.

7. Conclusions. - The Coulomb correlation corrections are responsible for the central dip in the otherwise nearly parabolic variation of the surface tension in the transitional series. They are not responsible for the similar central dip in the variation of the cohesive energy, which is due to the special stability of the free magnetic atoms with a half full $\mathrm{d}$ shell. These conclusions are in qualitative agreement with the results of Sayers' study of an s band in the Gutzwiller approximation [12]; and possibly with Kajzar's and Mizia's study in the Hubbard approximation [18]. The Coulomb correlation corrections provide a supplementary cohesion which peaks sinusoidally through the filling of a band. This does not alter the general behaviour of cohesion, but modifies strongly the Stoner criterion for ferromagnetism. It seems therefore that this correction must be taken into account in any detailed discussion of magnetism.

Appendix : Evaluation of numerical coefficients in the second-order term in $\boldsymbol{U}$. - Let $u_{l m}$ be the Coulomb potential energy between two electrons $l, m$ on the same atom.

When developing the second-order terms in the energy, one finds terms of the form

$$
\left|\left\langle v_{l}(l) v_{m}(m)\left|u_{l m}\right| w_{l}(l) w_{m}(m)\right\rangle\right|^{2}
$$

where $v_{l}, v_{m}$ are occupied $\mathrm{d}$ spin orbitals and $w_{l}$, $w_{m}$ unoccupied ones on the same atom. As $u_{l m}$ is spin independent, $v_{l}$ has the same spin as $w_{l}$, and $v_{m}$ the same as $w_{m}$. Of the four possible orbital integrals (A.1), we retain only the Coulomb integrals

$$
U_{l m}=\left\langle v_{l}(l) v_{m}(m)\left|u_{l m}\right| v_{l}(l) v_{m}(m)\right\rangle
$$

and assume them all to be equal to their average value $U$ :

$$
U_{l m} \simeq \bar{U}_{m}=U
$$

A.1 Paramagnetic metals. - Each atomic spin orbital has a probability $z / 10$ of being occupied and $1-z / 10$ of being empty. A given $U_{l m}^{2}$ thus occurs with a probability

$$
\left[\frac{z}{10}\left(1-\frac{z}{10}\right)\right]^{2} \text {. }
$$

The number of different $U_{l m}^{2}$ is

$$
\frac{10 \times 9}{2}=45 \text {, }
$$

for there are 10 different atomic spin-orbitals. Owing to the Pauli principle, they can only make a virtual collision with one of the 9 different spin-orbitals. Collisions of the pairs $l m$ and $m l$ are the same physical event and must not be counted twice.

Hence equation (6).

A.2 Ferromagnetic metals. - The average numbers $p, n$ per atom of electrons with positive and negative spins are defined as in the text.

A given $U_{l m}^{2}$ occurs with a probability

$$
\begin{gathered}
{\left[\frac{p}{5}\left(1-\frac{p}{5}\right)\right] \quad \text { for two } p \text { electrons }} \\
{\left[\frac{n}{5}\left(1-\frac{n}{5}\right)\right]^{2} \quad \text { for two } n \text { electrons }} \\
2 \frac{p}{5} \frac{n}{5}\left(1-\frac{p}{5}\right)\left(1-\frac{n}{5}\right)
\end{gathered}
$$

for one $p$ and one $n$ electrons.

The numbers of different $U_{l m}^{2}$ are, on the other hand,

$\frac{4 \times 5}{2}=10 \quad$ for two electrons of parallel spins

$\frac{5 \times 5}{2}=\frac{25}{2}$ for two electrons of opposites spins.

Thus, with the notation of equation (14),

$$
\begin{aligned}
\delta E^{2}(z, \mu)= & \frac{20 U^{2}}{2 w} \times \\
& \times\left\{\left[\frac{p}{5}\left(1-\frac{p}{5}\right)\right]^{2}+\left[\frac{n}{5}\left(1-\frac{n}{5}\right)\right]^{2}\right\} \\
& +\frac{25 U^{2}}{2 w} 2 \frac{p}{5} \frac{n}{5}\left(1-\frac{p}{5}\right)\left(1-\frac{n}{5}\right) \\
& -\frac{45 U^{2}}{w}\left[\frac{z}{10}\left(1-\frac{z}{10}\right)\right]^{2} .
\end{aligned}
$$

Replacing $p$ and $n$ in terms of $z$ and $\mu$ leads to equation (17). 


\section{References}

[1] FrIEDel, J., In Physics of Metals I, Electrons, Ed. J. M. Ziman (Cambridge University Press) 1969.

[2] Julg, M., Physique des petits grains, Colloque C.N.R.S. Lyon 1976, J. Physique Colloq. 38 (1977) C2.

[3] Friedel, J., Proc. Phys. Soc. B 65 (1952) 769.

[4] Slater, J. C., Phys. Rev. 82 (1951) 538.

[5] Cyrot-lackmann, F., Ducastelle, F. and Friedel, J., Solid State Commun. 8 (1970) 685.

[6] Brooks, H., Phys. Rev. 58 (1940) 909;

Callaway, J., Phys. Rev. 120 (1960) 731 ;

Lenglart, P., Thèse Paris (1967), Annls de Phys. (1968).

[7] Cf. Kittel, C., Introduction to Solid State Physics (Wiley New York) 1966.

[8] Fletcher, G. C. and Wohlfarth, E. P., Phil. Mag. 42 (1951) 106 ;

Asdente, M. and FrIedel, J., Phys. Rev. 124 (1961) 384; 126 (1962) 2262.
[9] Desjonquères, M. C. and Cyrot-Lackmann, private communication : DesJonquères, M. C., Thèse Grenoble (1976).

[10] Friedel, J., Annls de Phys. 1 (1976) 257.

[11] See for instance Gelatt, Jr., C. D., EhrenReich, H. and Watson, R. E., to be published Phys. Rev. 15 (1977).

[12] SAYERS, C. M., J. Phys. F., Physics of Metals under press (1977).

[13] Gutzwiller, M. C., Phys. Rev. 137A (1965) 1726.

[14] Kanamori, J., Prog. Theor. Phys. 30 (1963) 275; cf. also [1].

[15] Cyrot-Lackmann, F., Adv. Phys. 16 (1967) 393.

[16] LACOUR-GaYeT, P., Thèse Orsay (1974).

[17] Stoner, E. C., Rep. Progr. Phys. 9 (1946-7) 43.

[18] Kajzar, F. and Mizia, J., J. Phys. F., Physics of Metal under press (1977).

[19] GschneIDer, K. A., Solid State Phys. 16 (1964) 275.

[20] Friedel, J., J. Physique Radium 16 (1955) 829. 